Tôhoku Math. Journ.

25 (1973), 487-498.

\title{
SOME CRITICAL MAPPINGS
}

\author{
Dedicated to Professor Shigeo Sasaki on his 60th birthday
}

Yosio Mutō

(Received February 17, 1973)

Let $(N, h)$ and $(M, g)$ be connected compact orientable Riemannian manifolds of dimension $n$ and $m$ respectively, where $n \geqq m$. Let $\mu$ be a differentiable mapping of rank $m$ everywhere. There exists on $M$ an $m$-form $\varphi$ naturally induced from the metric $g$. The pull back $\psi$ of $\varphi$ is a closed $m$-form on $N$ and the integral

$$
J[\mu]=\int_{N}(\psi, \psi) d V_{N}
$$

is a functional of the mapping $\mu$. A critical point $\bar{\mu}$ of $J[\mu]$ is called in the present paper a critical mapping. The purpose of the present paper is to study some properties of such critical mappings.

Let $\left(M_{1}, f\right),\left(M_{2}, g\right)$, and $\left(M_{3}, h\right)$ be connected compact orientable Riemannian manifolds where $\operatorname{dim} M_{1}=\operatorname{dim} M_{2} \leqq \operatorname{dim} M_{3}$. If $\mu_{12}: M_{2} \rightarrow M_{1}$ and $\mu_{23}: M_{3} \rightarrow M_{2}$ are critical mappings, then $\mu_{13}=\mu_{12} \mu_{23}$ is a critical mapping of $M_{3}$ onto $M_{1}$. If a critical mapping $\mu$ is homeomorphic, its inverse $\mu^{-1}$ is also a critical mapping. When a set of Riemannian manifolds $\left\{\left(M_{\lambda}, g_{\lambda}\right), \lambda \in \Lambda\right\}$ of the same dimension is given where each manifold is connected, compact and orientable, the set of homeomorphic critical mappings forms a groupoid. Some examples of critical mappings are also given.

1. The functional $J[\mu]$ and its critical point. Let us consider connected compact orientable Riemannian manifolds $(M, g)$ and $(N, h)$ of dimension $m$ and $n$ respectively, such that $n \geqq m$ and admitting differentiable mappings $\mu: N \rightarrow M$ of rank $m$ everywhere. Local coordinates in $M$ are denoted by $x^{h}$ and those in $N$ by $y^{k}$. We use indices $h, i, j, \cdots=$ $1, \cdots, m$ for $M$ and indices $\kappa, \lambda, \mu, \cdots=1, \cdots, n$ for $N$. The metric tensors of $M$ and $N$ are denoted by $g_{j i}$ and $h_{\mu \lambda}$ respectively. When a mapping $\mu$ is expressed locally by $x^{h}=x^{h}\left(y^{1}, \cdots, y^{n}\right)$, we get connecting tensors

$$
B_{\kappa}^{h}=\frac{\partial x^{h}}{\partial y^{\kappa}}, \quad B_{\kappa_{1} \cdots \kappa_{p}}^{h_{1} \cdots h_{p}}=B_{\kappa_{1}}^{h_{1}} \cdots B_{\kappa_{p}}^{h_{p}}
$$


of the mapping.

The $m$-form

$$
\sqrt{\operatorname{det}\left(g_{j i}\right)} d x^{1} \cdots d x^{m}
$$

is a closed form on $M$ and

$$
B_{\lambda_{1} \cdots \lambda_{m}}^{[1 \cdots m} \sqrt{\operatorname{det}\left(g_{j i}\right)} d y^{\lambda_{1}} \cdots d y^{\lambda_{m}}
$$

is its pull back with respect to the mapping $\mu$. From this $m$-form let us define a functional $J[\mu]$ by

$$
\begin{aligned}
& J[\mu]=\int_{N} B_{\mu_{1} \cdots \mu_{m}}^{[1, \cdots m]} B_{\lambda_{1} \cdots \lambda_{m}}^{[1, \cdots m]} h^{\mu_{1} \lambda_{1}} \cdots h^{\mu_{m} \lambda_{m}} \operatorname{det}\left(g_{j i}\right) d V_{N}, \\
& d V_{N}=\sqrt{\operatorname{det}\left(h_{\mu \lambda}\right)} d y^{1} \cdots d y^{n} .
\end{aligned}
$$

Let us consider the space $\mathfrak{M}=\mathfrak{M}(N, M)$ of all such mappings $\mu$. Let $\mu_{0}$ be a point of $\mathfrak{M}$ and let $\mathscr{C}:[0,1] \rightarrow \mathfrak{M}$ be a curve $\{\mu(t), 0 \leqq t \leqq 1\}$ in $\mathfrak{M}$ where $\mu(0)=\mu_{0}$ and $\mu(t)$ is expressed locally by differentiable functions $x^{h}\left(y^{1}, \cdots, y^{n} ; t\right)$. For this curve $\mathscr{C}$ we define $\eta^{h}=D x^{h}$ by

$$
D x^{h}=\left(\frac{\partial x^{h}}{\partial t}\right)_{t=0}
$$

and $D J[\mu]$ by

$$
D J[\mu]=\left(\frac{d J[\mu(t)]}{d t}\right)_{t=0} .
$$

If $\mu_{0}$ is such that for all such curves $\mathscr{C} J[\mu]$ vanishes, then $\mu_{0}$ is a critical point $\bar{\mu}$ of the functional $J[\mu]$ defined by (1.1).

A critical point of $J[\mu]$ is called a critical mapping with respect to the integral $J[\mu]$. Let us define a necessary and sufficient condition of a critical mapping $\mu$ in tensor form.

For this purpose we use the connecting tensor $H_{\mu \lambda}{ }^{h}$ defined as the van der Waerden-Bortolotti derivative of $B_{\lambda}^{h}$, namely

$$
H_{\mu \lambda}{ }^{h}=\partial_{\mu} B_{\lambda}^{h}+\left\{\begin{array}{c}
h \\
j i
\end{array}\right\} B_{\mu i \lambda}^{j i}-\left\{\begin{array}{c}
\kappa \\
\mu \lambda
\end{array}\right\} B_{\kappa}^{h}
$$

where $\left\{\begin{array}{c}h \\ j i\end{array}\right\}$ and $\left\{\begin{array}{c}\kappa \\ \mu \lambda\end{array}\right\}$ are the Christoffels of $g_{j i}$ and $h_{\mu \lambda}$ respectively.

As we have

$$
D J[\mu]=\int_{N} D\left[B_{\mu_{1} \cdots \mu_{m}}^{[1, \cdots m]} B_{\lambda_{1} \cdots \lambda_{m}}^{\left[1 \cdots \lambda_{m}^{m}\right.} h^{\mu_{1} \lambda_{1} \cdots h^{\prime}} h^{\mu_{m} \lambda_{m}} \operatorname{det}\left(g_{j i}\right)\right] d V_{N}
$$

and 


$$
\begin{aligned}
D B_{k}^{h} & =\partial_{k} \eta^{h}, \\
D B_{\mu_{1} \cdots \mu_{m}}^{[1 \cdots \cdots]} & =m\left(D B_{\left[\mu_{1}\right.}^{[1}\right) B_{\left.\mu_{2} \cdots \mu_{m}\right]}^{2 \cdots m]} \\
& =m\left(\partial_{\left[\mu_{1}\right.} \eta^{[1}\right) B_{\left.\mu_{2} \cdots \mu_{m}\right]}^{2 \cdots \cdots}, \\
D g & =g g^{j i} \eta^{k} \partial_{k} g_{j i},
\end{aligned}
$$

where $g=\operatorname{det}\left(g_{j i}\right)$, we get

$$
\begin{aligned}
& D J[\mu]=\int_{N}\left[2 m\left(\partial_{\left[\mu_{1}\right.} \eta^{[1}\right) B_{\left.\mu_{2} \cdots \mu_{m}\right]}^{2 \cdots m]} B_{\lambda_{1} \cdots \lambda_{m}}^{[1 \cdots m]} h^{\mu_{1} \lambda_{1}} \cdots h^{\mu_{m} \lambda_{m}} g\right. \\
& \left.+B_{\mu_{1} \cdots \mu_{m}}^{[1, \cdots m]} B_{\lambda_{1} \cdots \lambda_{m}}^{[1 \cdots m]} h^{\mu_{1} \lambda_{1}} \cdots h^{\mu_{m} \lambda_{m}} g g^{j i} \eta^{k} \partial_{k} g_{j i}\right] d V_{N} \text {. }
\end{aligned}
$$

Since $\partial_{\left[\mu_{1}\right.} B_{\left.\mu_{2} \cdots \mu_{m}\right]}^{2 \cdots m}$ always vanish, we get from the above expression

$$
D J[\mu]=\int_{N} F d V_{N}
$$

where

$$
\begin{aligned}
& F=-2 m \eta^{[1} B_{\left[\mu_{2} \cdots \mu_{m}\right.}^{2 \cdots m]}\left(\partial_{\left.\mu_{1}\right]} B_{\lambda_{1} \cdots \lambda_{m}}^{[1, \cdots m]}\right) h^{\mu_{1} \lambda_{1}} \cdots h^{\mu_{m} \lambda_{m}} g \\
& -2 m \eta^{[1} B_{\left[\mu_{2} \cdots \mu_{m}\right.}^{2 \cdots m]}\left(\partial_{\left.\mu_{1}\right]}\left(h^{\mu_{1} \lambda_{1}} \cdots h^{\mu_{m} \lambda_{m}}\right)\right) B_{\left[\lambda_{1} \cdots \lambda_{m}\right]}^{[1 \cdots m]} g \\
& -2 m \eta^{[1} B_{\left[\mu_{2} \cdots \mu_{m}\right.}^{2 \cdots m]} B_{\left.\mu_{1}\right]}^{k} g^{j i}\left(\partial_{k} g_{j i}\right) B_{\lambda_{1} \cdots \lambda_{m}}^{[1 \cdots m]} h^{\mu_{1} \lambda_{1}} \cdots h^{\mu_{m} \lambda_{m}} g \\
& \text { - } m \eta^{[1} B_{\left[\mu_{2} \cdots \mu_{m}\right.}^{2 \cdots m]}\left(\partial_{\left.\mu_{1}\right]} h_{\omega \nu}\right) h^{\omega \nu} B_{\lambda_{1} \cdots \lambda_{m}}^{[1 \cdots m]} h^{\mu_{1} \lambda_{1}} \cdots h^{\mu_{m} \lambda_{m}} g \\
& +B_{\mu_{1} \cdots \mu_{m}}^{[1 \cdots m]} B_{\lambda_{1} \cdots \lambda_{m}}^{[1 \cdots]} h^{\mu_{1} \lambda_{1}} \cdots h^{\mu_{m} \lambda_{m}} g g^{j i} \eta^{k} \partial_{k} g_{j i} \text {. }
\end{aligned}
$$

On the other hand, we have

$$
\begin{aligned}
\partial_{\mu} B_{\lambda_{1} \cdots \lambda_{m}}^{[1 \ldots m]}= & \partial_{\mu} B_{\left[\lambda_{1} \cdots \lambda_{m}\right]}^{[1 \ldots m]} \\
= & m\left(\partial_{\mu} B_{\left[\lambda_{1}\right.}^{[1}{ }^{[1} B_{\left.\lambda_{2} \cdots \lambda_{m}\right]}^{2 \cdots m]}\right. \\
= & m H_{\mu\left[\lambda_{1}\right.}^{\left[{ }^{1} B_{\left.\lambda_{2} \cdots \lambda_{m}\right]}^{2} \cdots m\right]}+m\left\{\begin{array}{c}
\kappa \\
\mu\left[\lambda_{1}\right.
\end{array}\right\} B_{\left.[\kappa] \lambda_{2} \cdots \lambda_{m}\right]}^{[12 \cdots m]} \\
& -B_{\mu}^{k}\left\{\begin{array}{c}
j \\
k j
\end{array}\right\} B_{\lambda_{1} \cdots \lambda_{m}}^{[1 \ldots m]}
\end{aligned}
$$

where we have used (1.2) and the identity

$$
m\left\{\begin{array}{l}
{[1} \\
k j
\end{array}\right\} B_{\left[\lambda_{1} \cdots \lambda_{m}\right]}^{[j[2 \cdots m]}=\left\{\begin{array}{c}
j \\
k j
\end{array}\right\} B_{\left[\lambda_{1} \cdots \lambda_{m}\right]}^{[1 \ldots m]} .
$$

We also have

$$
\begin{aligned}
& m \eta^{[1} B_{\left[\mu_{2} \cdots \mu_{m}\right.}^{2 \cdots m]} B_{\left.\mu_{1}\right]}^{k} \\
& =\eta^{1} B_{\left[\mu_{2} \cdots \mu_{m}\right.}^{2 \cdots m} B_{\left.\mu_{1}\right]}^{k}-\sum_{t=2}^{m} \eta^{t} B_{\left[\mu_{2} \cdots \mu_{m}\right.}^{\left[2 \cdots \mu_{m} \cdots\right]} B_{\left.\mu_{1}\right]}^{k} \\
& =\eta^{k} B_{\mu_{1} \cdots \mu_{m}}^{[1 \cdots \cdots m]} .
\end{aligned}
$$

Substituting these identities into the expression of $F$ we get 


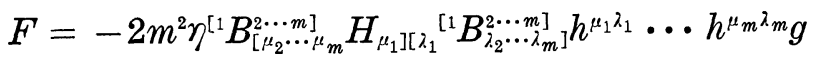

$$
\begin{aligned}
& -2 m^{2} \eta^{[1} B_{\left[\mu_{2} \cdots \mu_{m}\right.}^{2 \cdots m]}\left\{\begin{array}{c}
\kappa \\
\left.\mu_{1}\right]\left[\lambda_{1}\right.
\end{array}\right\} B_{\left.|\kappa| \lambda_{2} \cdots \lambda_{m}\right]}^{[12 \cdots \cdots]} h^{\mu_{1} \lambda_{1}} \cdots h^{\mu_{m} \lambda_{m}} g \\
& +2 \eta^{k} B_{\mu_{1} \cdots \mu_{m}}^{[1 \cdots m]}\left\{\begin{array}{c}
j \\
k j
\end{array}\right\} B_{\lambda_{1} \cdots \lambda_{m}}^{[1 \cdots m]} h^{\mu_{1} \lambda_{1}} \cdots h^{\mu_{m} \lambda_{m}} g \\
& -2 m^{2} \eta^{[1} B_{\left[\mu_{2} \cdots \mu_{m}\right.}^{2 \cdots m]}\left(\partial_{\left.\mu_{1}\right]} h^{\mu_{1} \lambda_{1}}\right) h^{\mu_{2} \lambda_{2}} \cdots h^{\mu_{m} \lambda_{m}} B_{\left[\lambda_{1} \cdots \lambda_{m}\right]}^{[1 \cdots m]} g \\
& -B_{\left[\mu_{1} \cdots \mu_{m}\right]}^{[1 \cdots m]} B_{\lambda_{1} \cdots \lambda_{m}}^{[1 \cdots m]} h^{\mu_{1} \lambda_{1}} \cdots h^{\mu m}{ }^{2} m g^{j i} \eta^{k}\left(\partial_{k} g_{j i}\right) g \\
& -2 m \eta^{[1} B_{\left[\mu_{2} \cdots \mu_{m}\right.}^{2 \cdots m]}\left\{\begin{array}{c}
\omega \\
\left.\mu_{1}\right] \omega
\end{array}\right\} B_{\lambda_{1} \cdots \lambda_{m}}^{[1 \cdots m]} h^{\mu_{1} \lambda_{1}} \cdots h^{\mu_{m} \lambda_{m}} g \text {. }
\end{aligned}
$$

Since we have

$$
\begin{aligned}
& \left\{\begin{array}{c}
\kappa \\
\mu\left[\lambda_{1}\right.
\end{array}\right\} B_{\left.|\kappa| \lambda_{2} \cdots \lambda_{m}\right]}^{[1 \cdots m]} h^{\left[\mu_{1}\left|\lambda_{1}\right| \cdots h^{\mu} \cdots \lambda_{m}\right.} \\
& \left.=\left\{\begin{array}{c}
\lambda_{1} \\
\mu \alpha
\end{array}\right\} B_{\lambda_{1} \cdots \lambda_{m}}^{[1 \cdots m]} h^{\left[\mu_{1}|\alpha|\right.} h^{\mu_{2}\left|\lambda_{2}\right|} \cdots h^{\mu}\right] \lambda_{m} \\
& \left.=\left\{\begin{array}{c}
\lambda_{1} \\
\mu \alpha
\end{array}\right\} B_{\left[\lambda_{1} \cdots \lambda_{m}\right]}^{[1 \cdots m]} h^{\left[\mu_{1}|\alpha|\right.} h^{\mu_{2}\left|\lambda_{2}\right|} \cdots h^{\mu} h_{m}\right]
\end{aligned}
$$

and $\partial_{\nu} h^{\mu \lambda}=-\left\{\begin{array}{c}\mu \\ \nu \alpha\end{array}\right\} h^{\alpha \lambda}-\left\{\begin{array}{c}\lambda \\ \nu \alpha\end{array}\right\} h^{\mu \alpha}$, we get

$$
\begin{aligned}
& -2 m^{2} \eta^{[1} B_{\left[\mu_{2} \cdots \mu_{m}\right.}^{2 \cdots m]}\left\{\begin{array}{c}
\kappa \\
\left.\mu_{1}\right]\left[\lambda_{1}\right.
\end{array}\right\} B_{\left[\kappa \mid \lambda_{2} \cdots \lambda_{m}\right]}^{[12 \cdots m]} h^{\mu_{1} \lambda_{1}} \cdots h^{\mu_{m} \lambda_{m}} \\
& -2 m^{2} \eta^{[1} B_{\left[\mu_{2} \cdots m\right]}^{2 \cdots m}\left(\partial_{\left.\mu_{1}\right]} h^{\mu_{1}\left[\lambda_{1}\right.}\right) h^{\left|\mu_{2}\right| \lambda_{2}} \cdots h^{\mid \mu} m^{\left.\mid \lambda_{m}\right]} B_{\left[\lambda_{1} \cdots \lambda_{m}\right]}^{[1 \cdots m]} \\
& =-2 m^{2} \eta^{[1} B_{\left[\mu_{2} \cdots \mu_{m}\right.}^{2 \cdots m]}\left\{\begin{array}{c}
\lambda_{1} \\
\left.\mu_{1}\right] \alpha
\end{array}\right\} B_{\left[\lambda_{1} \cdots \lambda_{m}\right]}^{[1 \cdots \cdots]} h^{\mu_{1} \alpha} h^{\mu_{2} \lambda_{2}} \cdots h^{\prime \prime} m^{2} m \\
& +2 m^{2} \eta^{[1} B_{\left[\mu_{2} \cdots \mu_{m}\right.}^{2 \cdots m]}\left\{\begin{array}{c}
\mu_{1} \\
\left.\mu_{1}\right] \alpha
\end{array}\right\} h^{\alpha \lambda_{1}} h^{\mu_{2} \lambda_{2}} \cdots h^{\mu_{m} \lambda_{m}} B_{\left[\lambda_{1} \cdots \lambda_{m}\right]}^{[1 \ldots m]} \\
& +2 m^{2} \eta^{[1} B_{\left[\mu_{2} \cdots \mu_{m}\right.}^{2 \cdots m]}\left\{\begin{array}{c}
\lambda_{1} \\
\left.\mu_{1}\right] \alpha
\end{array}\right\} h^{\mu_{1} \alpha} h^{\mu_{2} \lambda_{2}} \cdots h^{\mu_{m} m_{m}} B_{\left[\lambda_{1} \cdots \lambda_{m}\right]}^{[1 \cdots m]} \\
& =2 m^{2} \eta^{[1} B_{\left[\mu_{2} \cdots m\right]}^{2 \cdots \mu_{m}}\left\{\begin{array}{c}
\mu_{1} \\
\left.\mu_{1}\right] \alpha
\end{array}\right\} h^{\alpha \lambda_{1}} h^{\mu_{2} \lambda_{2}} \cdots h^{\mu_{m} \lambda_{m}} B_{\left[\lambda_{1} \cdots \lambda_{m}\right]}^{[1 \cdots \cdots]} \\
& =2 m \eta^{[1} B_{\left[\mu_{2} \cdots \mu_{m}\right]}^{2 \cdots m]}\left\{\begin{array}{c}
\omega \\
\omega \alpha
\end{array}\right\} h^{\alpha \lambda_{1}} h^{\mu_{2} \lambda_{2}} \cdots h^{\mu_{m}{ }_{m}} B_{\left[\lambda_{1} \cdots \lambda_{m}\right]}^{[1 \cdots m]} \\
& -2 m \sum_{t=2}^{m} \eta^{[1} B_{\left[\mu_{2} \cdots m\right]}^{\left.2 \cdots \mu_{1} \cdots \mu_{m}\right]}\left\{\begin{array}{c}
\mu_{1} \\
\mu_{t} \alpha
\end{array}\right\} h^{\alpha \lambda_{1}} h^{\mu_{2} \lambda_{2}} \cdots h^{\mu_{m} \lambda_{m}} B_{\left[\lambda_{1} \cdots \lambda_{m}\right]}^{[1 \cdots m]} .
\end{aligned}
$$


But the second term in the last member vanishes because of $\left\{\begin{array}{c}\mu_{1} \\ \mu_{t} \alpha\end{array}\right\}=\left\{\begin{array}{c}\mu_{1} \\ \alpha \mu_{t}\end{array}\right\}$ and we get

$$
2 m \eta^{[1} B_{\left[\mu_{2} \cdots \mu_{m}\right.}^{2 \cdots m]}\left\{\begin{array}{c}
\omega \\
\left.\mu_{1}\right] \omega
\end{array}\right\} h^{\mu_{1} \lambda_{1}} \cdots h^{\mu_{m} \lambda_{m}} B_{\left[\lambda_{1} \cdots \lambda_{m}\right]}^{[1 \cdots m]} g
$$

as the sum of the second and the fourth terms in the expression of $F$ in (1.3). This cancels the last term. Moreover, it is easy to see that the third term cancels the fifth term.

Hence we have

$$
F=-2 m^{2} \eta^{[1} B_{\left[\mu_{2} \cdots \mu_{m}\right.}^{2 \cdots m^{]}} H_{\left.\mu_{1}\right]\left[\lambda_{1}\right.}^{\left[{ }^{1}\right.} B_{\left.\lambda_{2} \cdots \lambda_{m}\right]}^{2 \cdots m]} h^{\mu_{1} \lambda_{1}} \cdots h^{\mu_{m}{ }_{m}} g \text {. }
$$

A critical mapping is characterized by the vanishing of $F$ for all $\eta^{k}$. Thus $\mu$ is a critical mapping if and only if

$$
\eta^{\left[j_{1}\right.} B_{\mu_{2} \cdots \mu_{m}}^{\left.j_{2} \cdots j_{m}\right]} H_{\mu_{1} \lambda_{1}}^{\left[i_{1}\right.} B_{\lambda_{2} \cdots \lambda_{m}}^{\left.i_{2} \cdots i_{m}\right]} h^{\left[\mu _ { 1 } \left[\lambda_{1}\right.\right.} \cdots h^{\left.\left.\mu_{m}\right] \lambda_{m}\right]}=0
$$

is satisfied by every vector field $\eta^{h}$ of $M$.

Let us define $H^{j i h}, H^{h}, h^{j i}$ and ' $h_{j i}$ by

$$
\begin{gathered}
H^{j i h}=B_{\mu \lambda}^{j i} H^{\mu \lambda h}=B_{\omega \nu}^{j i} h^{\omega \mu} h^{\nu \lambda} H_{\mu \lambda}{ }^{h}, \quad H^{h}=H_{\mu \lambda}{ }^{h} h^{\mu \lambda}=H^{\omega}{ }_{\omega}{ }^{h}, \\
'{ }^{j i}=B_{\mu \lambda}^{j i} h^{\mu \lambda}, \quad{ }^{\prime} h_{j i}{ }^{\prime} h^{j h}=\delta_{i}^{h} .
\end{gathered}
$$

As we have

$$
\begin{aligned}
& m \eta^{\left[j_{1}\right.} B_{\mu_{2} \cdots \mu_{m}}^{\left.j_{2} \cdots j_{m}\right]} H_{\mu_{1} \lambda_{1}}{ }^{\left[i_{1}\right.} B_{\lambda_{2} \cdots \lambda_{m}}^{\left.i_{2} \cdots i_{m}\right]} h^{\left[\mu _ { 1 } \left[\lambda_{1}\right.\right.} \cdots h^{\left.\left.\mu_{m}\right] \lambda_{m}\right]}=\eta^{\left[j_{1}\right.} B_{\mu_{2} \cdots \mu_{m}}^{\left.j_{2} \cdots j_{m}\right]} H_{\mu_{1} \lambda_{1}}{ }^{\left[i_{1}\right.} B_{\lambda_{2} \cdots \lambda_{m}}^{\left.i_{2} \cdots i_{m}\right]} \\
& \times\left(h^{\mu_{1} \lambda_{1}} h^{\mu_{2} \lambda_{2}} \cdots h^{\mu} \lambda_{m} \lambda_{i=2}^{m} h^{\mu_{1} \lambda_{t}} h^{\mu_{2} \lambda_{2}} \cdots h^{\mu_{t} \lambda_{1}} \cdots h^{\mu} \lambda_{m}\right),
\end{aligned}
$$

we can write (1.4) in the form

$$
\begin{aligned}
& \eta^{\left[j_{1}\right.} H^{\left[i_{1}\right.} h^{j_{2} i_{2}} \ldots{ }^{\prime} h^{\left.\left.j_{m}\right] i_{m}\right]} \\
& \quad-\sum_{t=2}^{m} \eta^{\left[j_{1}\right.} H^{\left[i_{t} j_{t} i_{1}{ }^{\prime}\right.} h^{j_{2} i_{2}} \ldots{ }^{\prime} h^{j_{t-1} i_{t-1}} h^{j_{t+1} i_{t+1}} \ldots{ }^{\prime} h^{\left.\left.j_{m}\right] i_{m}\right]}=0,
\end{aligned}
$$

hence

$$
\begin{aligned}
& \eta^{\left[j_{1}\right.} H^{\left[i_{1}\right.} h^{j_{2} i_{2}} \ldots h^{\prime} h^{\left.\left.j_{m}\right] i_{m}\right]} \\
& \quad+(m-1) \eta^{\left[j_{1}\right.} H^{\left[i_{1} j_{2} i_{2}\right.} h^{j_{3} i_{3}} \ldots h^{\prime} h^{\left.\left.j_{m}\right] i_{m}\right]}=0 .
\end{aligned}
$$

Since $i_{1}, \cdots, i_{m}$ and $j_{1}, \cdots, j_{m}$ run only the range $\{1, \cdots, m\}$, an equality of the form

$$
A^{\left[j _ { 1 } \left[i_{1} j_{2} i_{2} h^{j_{3} i_{3}} \cdots h^{\prime} h^{\left.\left.j_{m}\right] i_{m}\right]}\right.\right.}=0
$$

is equivalent to

$$
A^{j_{1} i_{1} j_{2} i_{2}}\left({ }^{\prime} h_{j_{1} i_{1}}{ }^{\prime} h_{j_{2} i_{2}}-{ }^{\prime} h_{j_{2} i_{1}}{ }^{\prime} h_{j_{1} i_{2}}\right)=0
$$


Hence we get from (1.5)

$$
\eta^{j} H^{i \prime} h_{j i}+\eta^{j}\left(H^{k l i}-H^{i l k}\right)^{\prime} h_{j k}{ }^{\prime} h_{l i}=0,
$$

and we can conclude that $\mu$ is a critical mapping if and only if $\mu$ satisfies

$$
H^{i}-H^{k j i \prime} h_{k j}+H^{i k j^{\prime}} h_{k j}=0 \text {. }
$$

Thus we have obtained the following theorem.

THEOREM 1.1. Let $(M, g)$ and $(N, h)$ be connected compact orientable Riemannian manifolds of dimension $m$ and $n$ respectively, where $n \geqq m$, and $\mu: N \rightarrow M$ be a differentiable mapping of rank $m$ everywhere. Then a necessary and sufficient condition for $\mu$ to be a critical mapping is that $\mu$ satisfies the equations

$$
H_{\mu \lambda}{ }^{h} h^{\mu \lambda}-H_{\omega \nu}{ }^{h} h^{\omega \mu} h^{\nu \lambda} B_{\mu \lambda}^{j i \prime} h_{j i}+H_{\omega \nu}{ }^{j} h^{\omega \mu} h^{\nu \lambda} B_{\mu \lambda}^{h k \prime} h_{k j}=0 \text {. }
$$

2. Some special cases. Let us consider the case where $\operatorname{dim} M=$ $\operatorname{dim} N=m$. Then we can define $B_{h}^{\kappa}$ by $B_{k}^{h} B_{i}^{\kappa}=\delta_{i}^{h}$ and get

$$
' h_{j i}=B_{j i}^{\mu \lambda} h_{\mu \lambda}, \quad B_{\mu \lambda}^{j i} h_{j i}=h_{\mu \lambda} \text {. }
$$

Hence we have in this special case

$$
H_{\omega \nu}{ }^{h} h^{\omega \mu} h^{\nu \lambda} B_{\mu \lambda}^{j i \prime} h_{j i}=H_{\omega \nu}{ }^{h} h^{\omega \nu} \text {. }
$$

This proves the following theorem.

TheOREM 2.1. Let $(M, g)$ and $(N, h)$ be connected compact orientable Riemannian manifolds of the same dimension $m$ and $\mu: N \rightarrow M$ be a differentiable mapping of rank $m$ everywhere. Then a necessary and sufficient condition for $\mu$ to be a critical mapping is that $\mu$ satisfies the equations

$$
H_{\mu \lambda}{ }^{h} B_{h}^{\lambda}=0 .
$$

Let us assume that there exists the inverse $\mu^{-1}: M \rightarrow N$ of $\mu$. Then $B_{h}^{\kappa}$ plays the same role in $\mu^{-1}$ as $B_{k}^{h}$ does in $\mu$ and the connecting tensor

$$
H_{j i}{ }^{\kappa}=\partial_{j} B_{i}^{\kappa}+\left\{\begin{array}{c}
\kappa \\
\mu \lambda
\end{array}\right\} B_{j i}^{\mu \lambda}-\left\{\begin{array}{c}
h \\
j i
\end{array}\right\} B_{h}^{\kappa}
$$

satisfies

$$
B_{\mu \lambda \lambda}^{j i h} H_{j i}{ }^{k}=-H_{\mu \lambda}{ }^{h} \text {. }
$$

This proves that $H_{j i}{ }^{k} B_{\kappa}^{i}$ vanishes if and only if $H_{\mu \lambda}{ }^{h} B_{h}^{\lambda}$ vanishes. Thus we have the 
COROLlaRY 2.2. If a differentiable mapping $\mu: N \rightarrow M$ admits the inverse mapping $\mu^{-1}: M \rightarrow N$, one is a critical mapping if and only if the other is a critical mapping.

Let us consider connected compact orientable Riemannian manifolds $\left(M_{1}, f\right),\left(M_{2}, g\right),\left(M_{3}, h\right)$ of the same dimension $m$ and assume that there exist critical mappings $\mu_{12}: M_{2} \rightarrow M_{1}$ and $\mu_{23}: M_{3} \rightarrow M_{2}$. Let us use $u^{\alpha}, x^{h}$ and $y^{\kappa}$ for the local coordinates in $M_{1}, M_{2}$, and $M_{3}$ respectively. For the mapping $\mu_{12}$ we have

$$
B_{h}^{\alpha}=\frac{\partial u^{\alpha}}{\partial x^{h}}, \quad H_{j i}{ }^{\alpha}=\partial_{j} B_{i}^{\alpha}+\left\{\begin{array}{c}
\alpha \\
\gamma \beta
\end{array}\right\} B_{j i}^{\gamma \beta}-\left\{\begin{array}{c}
h \\
j i
\end{array}\right\} B_{h}^{\alpha},
$$

and for the mapping $\mu_{23}$ we have

$$
B_{\kappa}^{h}=\frac{\partial x^{h}}{\partial y^{\kappa}}, \quad H_{\mu \lambda}{ }^{h}=\partial_{\mu} B_{\lambda}^{h}+\left\{\begin{array}{c}
h \\
j i
\end{array}\right\} B_{\mu \lambda}^{j i}-\left\{\begin{array}{c}
\kappa \\
\mu \lambda
\end{array}\right\} B_{\kappa}^{h},
$$

where $\left\{\begin{array}{c}\alpha \\ \gamma \beta\end{array}\right\},\left\{\begin{array}{c}h \\ j i\end{array}\right\}$, and $\left\{\begin{array}{c}\kappa \\ \mu \lambda\end{array}\right\}$ are the Christoffels derived from $f_{\gamma \beta}, g_{j i}$, and $h_{\mu \lambda}$ respectively. If we define $B_{\alpha}^{h}$ and $B_{h}^{\kappa}$ by

we have

$$
B_{\beta}^{h} B_{h}^{\alpha}=\delta_{\beta}^{\alpha}, \quad B_{\lambda}^{h} B_{h}^{\kappa}=\delta_{\lambda}^{\kappa},
$$

$$
H_{j i}{ }^{\alpha} B_{\alpha}^{i}=0, \quad H_{\mu \lambda}{ }^{h} B_{h}^{\lambda}=0 .
$$

Let us consider the mapping $\mu_{13}=\mu_{12} \mu_{23}$ of $M_{3}$ onto $M_{1}$. The connecting tensor of this mapping is

$$
B_{\kappa}^{\alpha}=\frac{\partial u^{\alpha}}{\partial y^{\kappa}}=B_{i}^{\alpha} B_{\kappa}^{i}
$$

and we get

hence

$$
\begin{aligned}
H_{\mu \lambda}{ }^{\alpha}= & \partial_{\mu} B_{\lambda}^{\alpha}+\left\{\begin{array}{c}
\alpha \\
\gamma \beta
\end{array}\right\} B_{\mu \lambda}^{\gamma \beta}-\left\{\begin{array}{c}
\kappa \\
\mu \lambda
\end{array}\right\} B_{\kappa}^{\alpha} \\
= & \left(B_{\mu}^{j} \partial_{j} B_{i}^{\alpha}\right) B_{\lambda}^{i}+B_{h}^{\alpha} \partial_{\mu} B_{\lambda}^{h} \\
& +\left\{\begin{array}{c}
\alpha \\
\gamma \beta
\end{array}\right\} B_{j i}^{\gamma \beta} B_{\mu \lambda}^{j i}-\left\{\begin{array}{c}
\kappa \\
\mu \lambda
\end{array}\right\} B_{h}^{\alpha} B_{\kappa}^{h}
\end{aligned}
$$

$$
H_{\mu \lambda}{ }^{\alpha}=H_{j i}{ }^{\alpha} B_{\mu \lambda}^{j i}+H_{\mu \lambda}{ }^{h} B_{h}^{\alpha} .
$$

Then we immediately obtain

$$
\begin{aligned}
H_{\mu \lambda}{ }^{\alpha} B_{\alpha}^{\lambda} & =H_{j i}{ }^{\alpha} B_{\mu \lambda}^{j i} B_{k}^{\lambda} B_{\alpha}^{k}+H_{\mu \lambda}{ }^{h} B_{h}^{\alpha} B_{k}^{\lambda} B_{\alpha}^{k} \\
& =B_{\mu}^{j} H_{j i}{ }^{\alpha} B_{\alpha}^{i}+H_{\mu \lambda}{ }^{h} B_{h}^{\lambda} \\
& =0
\end{aligned}
$$


by virtue of (2.2). This proves that, if $\mu_{12}$ and $\mu_{23}$ are critical mappings, then $\mu_{12} \mu_{23}$ is also a critical mapping.

Now let us consider a set of Riemannian manifolds $\left\{\left(M_{\lambda}, g_{\lambda}\right), \lambda \in \Lambda\right\}$ of the same dimension where each manifold is connected, compact and orientable. For any $\kappa, \lambda \in \Lambda$ we denote the set of homeomorphic critical mappings $M_{\kappa} \rightarrow M_{\lambda}$ by $G_{\lambda, \kappa}$. $G_{\lambda, \lambda}$ contains the identity mapping $e_{\lambda}: M_{\lambda} \rightarrow M_{\lambda}$, but $e_{\kappa}$ and $e_{\lambda}$ are distinguished if $\kappa \neq \lambda$. Then from the above results we see that the union of $G_{\lambda, \kappa}$ for all $\kappa, \lambda \in \Lambda$ forms a groupoid.

Thus we obtain the following theorem.

TheOREM 2.3. When a set of Riemannian manifolds $\left\{\left(M_{\lambda}, g_{\lambda}\right), \lambda \in \Lambda\right\}$ of the same dimension is given where each manifold is connected, compact and orientable, the set of homeomorphic critical mappings forms a groupoid.

In this theorem we have assumed that all Riemannian manifolds are of the same dimension. This assumption is essential. We consider now a case where $\mu_{12}: M_{2} \rightarrow M_{1}$ and $\mu_{23}: M_{3} \rightarrow M_{2}$ are critical mappings and $m_{1}=$ $\operatorname{dim} M_{1}, m_{2}=\operatorname{dim} M_{2}, m_{3}=\operatorname{dim} M_{3}$ satisfy $m_{1}=m_{2}<m_{3}$. Then we have (1.7) and $H_{j i}{ }^{\alpha} B_{\alpha}^{i}=0$. If we consider the mapping $\mu_{13}=\mu_{12} \mu_{23}$ we get (2.3) for this mapping too. Then we can prove that $\mu_{13}$ is also a critical mapping.

For this purpose we define $" h^{\gamma \beta}$ and " $h_{r \beta}$ by

$$
" h^{\gamma \beta}=B_{\mu \lambda}^{\gamma \beta} h^{\mu \lambda}, \quad " h_{\gamma \beta}{ }^{\prime \prime} h^{\gamma \alpha}=\delta_{\beta}^{\alpha} .
$$

Then we get

$$
\begin{aligned}
& H_{\mu \lambda}{ }^{\alpha} h^{\mu \lambda}=H_{j i}{ }^{\alpha} B_{\mu \lambda}^{j i} h^{\mu \lambda}+H_{\mu \lambda}{ }^{h} h^{\mu \lambda} B_{h}^{\alpha} \\
& =H_{j i}{ }^{\alpha} h^{j i}+H_{\mu \lambda}{ }^{h} h^{\mu \lambda} B_{h}^{\alpha}, \\
& -H_{\omega \nu}{ }^{\alpha} h^{\omega \mu} h^{\nu \lambda} B_{\mu \lambda}^{\gamma \beta \prime \prime} h_{\gamma \beta} \\
& =-\left(H_{j i}{ }^{\alpha} B_{\omega \nu}^{j i} h^{\omega \mu} h^{\nu \lambda}+H_{\omega \nu}{ }^{h} B_{h}^{\alpha} h^{\omega \mu} h^{\nu \lambda}\right) B_{l k}^{\gamma \beta} B_{\mu \lambda}^{l k \prime \prime} h_{\gamma \beta} \\
& =-H_{j i}{ }^{\alpha} h^{j{ }^{j \prime}} h^{i k^{\prime}} h_{l k}-H_{\omega \nu}{ }^{k} h^{\omega \mu} h^{\nu \lambda} B_{\mu_{\alpha}}^{l k^{\prime} \prime} h_{l k} B_{h}^{\alpha} \\
& =-H_{j i}{ }^{\alpha} h^{j i}-H_{\omega \nu}{ }^{h} h^{\omega \mu} h^{\nu \lambda} B_{\mu \lambda}^{j i} h_{j i} B_{h}^{\alpha}, \\
& H_{\omega \nu}{ }^{\gamma} h^{\omega \mu} h^{\nu \lambda} B_{\mu \lambda}^{\alpha, \prime \prime} h_{\varepsilon \gamma} \\
& =H_{l k}^{\gamma} B_{\omega \nu}^{l k} h^{\omega \mu} h^{\nu \lambda} B_{\mu \lambda}^{h j} B_{h j}^{\alpha \varepsilon \prime \prime} h_{\varepsilon r}+H_{\omega \nu}{ }^{j} B_{j}^{\gamma} h^{\omega \mu} h^{\nu \lambda} B_{\mu \lambda}^{h k} B_{h k}^{\alpha \varepsilon{ }^{\prime \prime}} h_{\varepsilon r} \\
& =H_{l k}{ }^{\gamma} h^{l h^{\prime}} h^{k j} B_{j}^{\varepsilon \prime \prime} h_{\varepsilon \gamma} B_{h}^{\alpha}+H_{\omega \nu}{ }^{j} h^{\omega \mu} h^{\nu \lambda} B_{\mu \lambda}^{h k \prime} h_{k j} B_{h}^{\alpha} \\
& =H_{l k}{ }^{\gamma} B_{r}^{k \prime} h^{l k} B_{h}^{\alpha}+H_{\omega \nu}{ }^{j} h^{\omega \mu} h^{\nu \lambda} B_{\mu \lambda}^{h h^{\prime}} h_{k j} B_{h}^{\alpha} \text {. }
\end{aligned}
$$

As the first term in the last member vanishes, we get

$$
H_{\mu \lambda}{ }^{\alpha} h^{\mu \lambda}-H_{\omega \nu}{ }^{\alpha} h^{\omega \mu} h^{\nu \lambda} B_{\mu \lambda}^{\gamma \beta \prime \prime} h_{\gamma \beta}+H_{\omega \nu}{ }^{\gamma} h^{\omega \mu} h^{\nu \lambda} B_{r \lambda \lambda}^{\alpha \varepsilon} h_{\varepsilon \gamma}=0
$$

by virtue of (1.7). 
Thus we have the following theorem.

THEOREM 2.4. Let there be three connected compact orientable Riemannian manifolds $\left(M_{1}, f\right),\left(M_{2}, g\right)$, and $\left(M_{3}, h\right)$ admitting critical mappings $\mu_{12}: M_{2} \rightarrow M_{1}$ and $\mu_{23}: M_{3} \rightarrow M_{2}$. If $\operatorname{dim} M_{1}=\operatorname{dim} M_{2}$ and $\operatorname{dim} M_{2}<\operatorname{dim} M_{3}$, the mapping $\mu_{13}=\mu_{12} \mu_{23}$ is a critical mapping.

If we want to prove only the Theorem 2.3 , we can use the following property of a critical mapping.

If $\operatorname{dim} N=\operatorname{dim} M=m$, the connecting tensor $B_{k}^{h}$ of a critical mapping $\mu:(N, h) \rightarrow(M, g)$ satisfies

$$
B_{\left[\kappa_{1} \cdots \kappa_{m}\right]}^{\left[h_{1} \ldots h_{m}\right]} \frac{\sqrt{g}}{\sqrt{h}}=\text { const. }
$$

Conversely, if (2.4) is satisfied, $\mu$ is a critical mapping.

(2.4) is proved by taking the partial derivatives and using (2.1).

3. Infinitesimal transformations of a Riemannian manifold. Let us take a one-parameter group of transformations $\mu(t)$ of a connected compact orientable Riemannian manifold $(M, g)$. Then we have a case of $N=M$, $h=g$. If $\mu(t)$ takes a point $P$ into $Q=\mu(t) P$ and the local coordinates of $P$ and $Q$ are respectively denoted by $x^{h}(P)$ and $x^{h}(Q), \partial x^{h}(Q) / \partial x^{i}(P)$ plays the role of $B_{k}^{h}$ and

$$
\frac{\partial^{2} x^{h}(Q)}{\partial x^{j}(P) \partial x^{i}(P)}+\left\{\begin{array}{c}
h \\
l k
\end{array}\right\}_{Q} \frac{\partial x^{l}(Q)}{\partial x^{j}(P)} \frac{\partial x^{k}(Q)}{\partial x^{i}(P)}-\left\{\begin{array}{c}
k \\
j i
\end{array}\right\}_{P} \frac{\partial x^{h}(Q)}{\partial x^{k}(P)}
$$

plays the role of $H_{\mu 2}{ }^{h}$. Hence the transformations $\mu(t)$ are critical mappings of $(M, g)$ onto $(M, g)$ if and only if

$$
\frac{\partial x^{i}(P)}{\partial x^{h}(Q)}\left[\frac{\partial^{2} x^{h}(Q)}{\partial x^{j}(P) \partial x^{i}(P)}+\left\{\begin{array}{c}
h \\
l k
\end{array}\right\}_{Q} \frac{\partial x^{l}(Q)}{\partial x^{j}(P)} \frac{\partial x^{k}(Q)}{\partial x^{i}(P)}-\left\{\begin{array}{c}
k \\
j i
\end{array}\right\}_{P} \frac{\partial x^{h}(Q)}{\partial x^{k}(P)}\right]=0
$$

is satisfied.

Let $v^{h}$ be a vector field on $M$ generating the group $\mu(t)$. Then we get

$$
\delta_{h}^{i}\left[\frac{\partial^{2} v^{h}}{\partial x^{j} \partial x^{i}}+\partial_{k}\left\{\begin{array}{c}
h \\
j i
\end{array}\right\} v^{k}+\left\{\begin{array}{c}
h \\
k i
\end{array}\right\} \partial_{j} v^{k}+\left\{\begin{array}{c}
h \\
j k
\end{array}\right\} \partial_{i} v^{k}-\left\{\begin{array}{c}
k \\
j i
\end{array}\right\} \partial_{k} v^{h}\right]=0
$$

from (3.1). But this is equivalent to

$$
\nabla_{j} \nabla_{i} v^{i}=0,
$$

hence $\nabla_{i} v^{i}=C$. On the other hand, we have always 


$$
\int_{M} \nabla_{i} v^{i} d V_{M}=0
$$

Hence we get

$$
\nabla_{i} v^{i}=0 \text {. }
$$

Thus we obtain the following theorem.

THEOREM 3.1. A one-parameter group of transformations of a connected compact orientable Riemannian manifold $(M, g)$ generated by a vector field $v^{h}$ is a group of critical mappings if and only if $v^{h}$ satisfies $\nabla_{i} v^{i}=0$. The set of all such vector fields forms a Lie algebra.

\section{Examples.}

$1^{\circ}$. Coclosed mappings. Let $\mu:(N, h) \rightarrow(M, g)$ be a coclosed mapping [1]. Then

$$
-H_{\omega \nu}{ }^{h} h^{\omega \mu} B_{\mu}^{i}+H_{\omega \nu}{ }^{i} h^{\omega \mu} B_{\mu}^{h}=B_{\nu}^{k} P_{k}{ }^{i h}, H_{\mu \lambda}{ }^{h} h^{\mu \lambda}=-P_{k}{ }^{k h}
$$

are compatible. From (4.1) we obtain

$$
-H_{\omega \nu}{ }^{h} h^{\omega \mu} h^{\nu \lambda} B_{\mu \lambda}^{i j \prime} h_{i j}+H_{\omega \nu}{ }^{i} h^{\omega \mu} h^{\nu \lambda} B_{\mu \lambda}^{h{ }^{\prime} h_{j i}} h_{j i}=P_{k}{ }^{i h} B_{\nu \lambda}^{k j} h^{\nu \lambda} h_{i j}=P_{k}{ }^{k h}=-H_{\mu \lambda}{ }^{h} h^{\mu \lambda} \text {, }
$$

which proves that $\mu$ is then a critical mapping.

A geodesic mapping is a mapping where $H_{\mu \lambda}{ }^{h}$ vanishes. Hence this is a coclosed mapping [1] and also a critical mapping.

$2^{\circ}$. A critical mapping $\mu:(N, h) \rightarrow(M, g)$ where $\operatorname{dim} N-\operatorname{dim} M=1$. In this case a vector field $\xi^{\kappa}$ of $(N, h)$ is determined by

$$
B_{\kappa}^{h} \xi^{\kappa}=1, \quad h_{\mu} \xi^{\mu} \xi^{\lambda}=1 \text {. }
$$

Let $\xi_{\lambda}$ be defined by $\xi_{\lambda}=h_{\lambda \kappa} \xi^{\kappa}$ and let $\left(B_{i}^{\kappa}, \xi^{\kappa}\right)$ be the inverse matrix of $\left(B_{\lambda}^{h}, \xi_{\lambda}\right)$, namely such that

$$
B_{i}^{\kappa} \xi_{\kappa}=0, \quad B_{i}^{\kappa} B_{\kappa}^{h}=\delta_{i}^{h} .
$$

Then we have

$$
{ }^{\prime} h_{j i}=B_{j i}^{\mu \lambda} h_{\mu \lambda}, \quad B_{i}^{\kappa} B_{\lambda}^{i}=\delta_{\lambda}^{\kappa}-\xi^{\kappa} \xi_{\lambda} \cdot
$$

The condition that $\mu$ is a critical mapping is written in the form

$$
H_{\mu \lambda}{ }^{h} h^{\mu \lambda}-H_{\omega \nu}{ }^{h} h^{\omega \mu} h^{\nu \lambda} B_{\mu \lambda}^{j i} B_{j i}^{\rho \sigma} h_{\rho \sigma}+H_{\omega \nu}{ }^{j} h^{\omega \mu} h^{\nu \lambda} B_{\mu \lambda}^{h k} B_{k j}^{\rho \sigma} h_{\rho \sigma}=0 \text {. }
$$

As we have

$$
H_{\omega \nu}{ }^{h} h^{\omega \mu} h^{\nu \lambda}\left(\delta_{\mu}^{\rho}-\xi_{\mu} \xi^{\rho}\right)\left(\delta_{\lambda}^{\sigma}-\xi_{\lambda} \xi^{\sigma}\right) h_{\rho \sigma}=H_{\omega \nu}{ }^{h} h^{\omega \nu}-H_{\omega \nu}{ }^{h} \xi^{\omega} \xi^{\nu}
$$

and

$$
H_{\omega \nu}{ }^{j} h^{\omega \mu} h^{\nu \lambda} B_{\mu}^{h} B_{j}^{o}\left(\delta_{\lambda}^{\rho}-\xi_{\lambda} \xi^{\rho}\right) h_{\rho \sigma}=H_{\omega \nu}{ }^{j} h^{\omega \mu} B_{\mu}^{h} B_{j}^{\nu},
$$


we get

$$
H_{\mu \lambda}{ }^{h} \xi^{\mu} \xi^{\lambda}+H_{\mu \lambda}{ }^{j} h^{\mu \kappa} B_{\kappa}^{h} B_{j}^{\lambda}=0
$$

as a necessary and sufficient condition of a critical mapping.

$3^{\circ}$. Projection of a fibred Riemannian manifold $\tilde{M}$ with an invariant Riemannian metric $h$ onto the base manifold $\left(M^{*}, g\right)$. Let $\tilde{M}$ be a fibred Riemannian manifold with $S^{1}$ as the type fibre and with an invariant Riemannian metric $h$ [2]. The base manifold $\left(M^{*}, g\right)$ is assumed to be a compact orientable Riemannian manifold. We denote the projection by $\pi$. For this mapping the vector field $\xi^{\kappa}$ determined by

$$
B_{\kappa}^{h} \xi^{\kappa}=0, \quad h_{\mu \lambda} \xi^{\mu} \xi^{\lambda}=1
$$

is a Killing vector field of $\tilde{M}$. If $\tilde{\nabla}$ denotes the covariant differentiation with respect to the metric $h$, we get $\tilde{\nabla}_{\mu} \xi_{\lambda}+\tilde{\nabla}_{\lambda} \xi_{\mu}=0$ and $\xi^{\mu} \tilde{\nabla}_{\mu} \xi^{\lambda}=0$.

As $h_{\mu \lambda}$ is an invariant metric, we have

$$
B_{\mu \lambda}^{j i} h^{\mu \lambda}=g^{j i} \text {. }
$$

We have also

$$
H_{\mu \lambda}{ }^{h} \xi^{\mu} \xi^{\lambda}=0
$$

by virtue of $B_{\kappa}^{h} \xi^{\kappa}=0$ and $\left(\xi^{\mu} \tilde{\nabla}_{\mu} \xi^{\lambda}\right) B_{\lambda}^{h}=0$.

On the other hand, applying van der Waerden-Bortolotti differentiation to $(4.3)$ we get

$$
H_{\nu \mu}^{j} B_{\lambda}^{i} h^{\mu \lambda}+H_{\nu \lambda}{ }^{i} B_{\mu}^{j} h^{\mu \lambda}=\nabla_{\nu} g^{j i}=0
$$

and consequently

$$
0=H_{\nu \mu}{ }^{j} B_{\lambda}^{i} h^{\mu \lambda} B_{j}^{\rho} B_{i}^{\sigma} h_{\rho \sigma}=H_{\nu \mu}{ }^{j} h^{\mu \lambda} B_{j}^{\rho} h_{\rho_{\sigma}}\left(\delta_{\lambda}^{\sigma}-\xi_{\lambda} \xi^{\sigma}\right)=H_{\nu \mu}{ }^{j} B_{j}^{\mu} \cdot
$$

Hence $\pi$ satisfies (4.2) and is a critical mapping among all mappings $\mu: \tilde{M} \rightarrow M^{*}$.

$4^{\circ}$. A critical mapping $\mu:(N, h) \rightarrow(M, g)$ where $\operatorname{dim} N=\operatorname{dim} M$. Let the local coordinates of $N$ and $M$ be chosen such that the point $P$ of $N$ and the point $\mu P$ of $M$ have the same coordinates $x^{h}$. Then we have

$$
B_{\kappa}^{h}=\delta_{\kappa}^{h}, \quad H_{j i}{ }^{h}=\left\{\begin{array}{c}
h \\
j i
\end{array}\right\}_{g}-\left\{\begin{array}{c}
h \\
j i
\end{array}\right\}_{h}
$$

where $\left\{\begin{array}{c}h \\ j i\end{array}\right\}_{g}$ and $\left\{\begin{array}{l}h \\ j i\end{array}\right\}_{h}$ are the Christoffels derived respectively from $g$ and $h$. From (2.1) we get 


$$
\left\{\begin{array}{c}
i \\
j i
\end{array}\right\}_{g}=\left\{\begin{array}{c}
i \\
j i
\end{array}\right\}_{h}
$$

as a necessary and sufficient condition for a critical mapping. This result also proves Theorem 2.3.

\section{REFERENCES}

[1] Y. Muтō, On coclosed mappings, Hokkaido Math. J. 1 (1972), 218-227.

[2] K. Yano AND S. IshIHARA, Fibred spaces with invariant Riemannian metric, Kōdai Math. Sem. Rep. 19 (1967), 317-360.

Department of APPlied Mathematics

YOKOHAMA NATIONAL UNIVERSITY

YOKOHAMA, JAPAN 